\title{
Verzeichnis der Bearbeiter der 6. Auflage
}

Professor Dr. Jochen Axer, Rechtsanwalt, Wirtschaftsprüfer, Steuerberater, axis Rechtsanwälte, Köln

Professor Dr. Jens-Hinrich Binder, LL.M. (London), Universität Tübingen

Professor Dr. Benjamin B. von Bodungen, LL.M. (Auckland), GGS, Heilbronn

Professor Dr. Jens Bülte, Universität Mannheim

Professor Dr. Ulrich Burgard, Otto-von-Guericke-Universität Magdeburg

Professor em. Dr. Dr. h.c. mult. Claus-Wilhelm Canaris, Ludwig-Maximilians-Universität München $\dagger$

Professor Dr. Matthias Casper, Westfälische Wilhelms-Universität Münster

Professor Dr. Klaus-Dieter Drüen, Ludwig-Maximilians-Universität München

Max Ehrl, Notarassessor, Geschäftsführer des Deutschen Notarvereins, Berlin

Dr. Raimond Emde, Rechtsanwalt, GvW Graf von Westphalen, Hamburg

Prof. Dr. Philipp S. Fischinger, Universität Mannheim

Jun.-Prof. Dr. Stephan Gräf, Universität Konstanz

Professor Dr. Hans Christoph Grigoleit, Ludwig-Maximilians-Universität München

Professor Dr. Dr. Stefan Grundmann, LL.M. (Berkeley), Humboldt-Universität zu Berlin und European University Institute in Florenz

Professor Dr. Mathias Habersack, Ludwig-Maximilians-Universität München

Professor Dr. Stephan Harbarth, LL.M. (Yale), Präsident des Bundesverfassungsgerichts

Professor Dr. Dr. h.c. mult. Peter Hommelhoff, Ruprecht-Karls-Universität Heidelberg

Professor Dr. Henning Jessen, LL.M. (Tulane), World Maritime University Malmö

Professor Dr. Christian Kersting, LL.M. (Yale), Heinrich-Heine-Universität Düsseldorf

Professor Dr. Peter Kindler, Ludwig-Maximilians-Universität München

Professor Dr. Detlef Kleindiek, Universität Bielefeld

Professor Dr. Jens Koch, Rheinische Friedrich-Wilhelms-Universität Bonn

Dr. Ernst-Thomas Kraft, Rechtsanwalt, Hengeler Mueller, Frankfurt am Main

Daniela Mattheus, Rechtsanwältin, Präsidentin der FEA, Berlin

Professor Dr. Andreas Maurer, LL.M. (Osgoode), Universität Mannheim

Professor Dr. André Meyer, LL.M. Taxation, Universität Bayreuth

Professor Dr. Florian Möslein, LL.M. (London), Phillips-Universität Marburg

Professor Dr. Hartmut Oetker, Christian-Albrechts-Universität, Kiel

Professor Dr. Karsten Otte, M.J.C. (Austin), außerplanmäßige Professur an der Universität Mannheim, Direktor bei der Bundesnetzagentur, Bonn

PD Dr. Moritz Pöschke, LL.M. (Harvard), Universität zu Köln, Rechtsanwalt, Dipl.-Kfm.

Professor Dr. Moritz Renner, Universität Mannheim

Dr. Fabian Reuschle, Richter am Landgericht Stuttgart

Professor Dr. Carsten Schäfer, Universität Mannheim

Professor Dr. Patrick Schmidt, Rechtsanwalt, NJP Grotstollen, Duisburg

Harald Schoen, LL.M., Referatsleiter BMJV Berlin

Professor Dr. Martin Schwab, Universität Bielefeld

Professor Dr. Jan Thiessen, Humboldt-Universität zu Berlin

PD Dr. Chris Thomale, LL.M. (Yale), Ruprecht-Karls-Universität Heidelberg

PD Dr. Andreas Weber, Ludwig-Maximilians-Universität München

Professor Dr. Christoph Weber, Julius-Maximilians-Universität Würzburg 
